\title{
Pregabalin reduces sleep disturbance in patients with generalized anxiety disorder via both direct and indirect mechanisms
}

\author{
Vamsi Bollu* \\ Andrew G Bushmakin** \\ Joseph C Cappelleri** \\ Chwen-Cheng Chen** \\ Douglas Feltner** \\ Hans-Ulrich Wittchen ${ }^{\star \star *}$ \\ * Novartis Pharmaceuticals Corporation, \\ Florham Park, New Jersey \\ ** Pfizer Inc, Global Research and \\ Development, New London \\ *** University of Dresden, Dresden \\ GERMANY
}

USA

\begin{abstract}
Background and Objectives: To characterize the impact of pregabalin on sleep in patients with generalized anxiety disorder (GAD) and to determine whether the impact is a direct or an indirect effect, mediated through the reduction of anxiety symptoms.

Methods: A post-hoc analysis of data from a randomized, double-blind, placebo- and active-controlled study in patients with GAD was conducted. Patients received pregabalin 300 $\mathrm{mg} /$ day, venlafaxine XR $75 \mathrm{mg}$ /day or placebo for a week, followed by pregabalin 300-600 $\mathrm{mg} /$ day, venlafaxine XR 75-225 mg/day, or placebo for 7 weeks. Treatment effect on sleep was evaluated using the Medical Outcomes Study Sleep Scale. Anxiety symptoms were assessed with the Hamilton Anxiety Rating Scale. A mediation model was used to estimate separately for both treatment arms the direct and indirect treatment effects on sleep disturbance.

Results: Compared with placebo $(\mathrm{n}=128)$, treatment with pregabalin $(\mathrm{n}=121)$ significantly reduced scores on the sleep disturbance subscale and Sleep Problems Index II at both week 4 and week 8 , and the sleep adequacy subscale at week 8 . Venlafaxine XR ( $\mathrm{n}=$ 125 ) had no significant effect on these measures. The mediation model indicated that $53 \%$ of the total pregabalin effect on sleep disturbance was direct $(p<0.01)$ and $47 \%$ indirect, mediated through anxiety symptoms $(p<0.05)$.

Conclusions: Pregabalin decreased sleep disturbance in patients with GAD both directly, and indirectly by reducing anxiety symptoms. Given the drug specificity of the results, this study provides evidence of an additional important pathway of action for pregabalin and its efficacy in GAD.
\end{abstract}




\section{Introduction}

Insomnia is one of the six core hyperarousal symptoms that characterize generalized anxiety disorder (GAD) according to the Diagnostic and Statistical Manual of Mental Disorders (Fourth Edition, Text Revision; DSM IV-TR) criteria. More than two-thirds of patients suffer from one or more forms of sleep disturbances ${ }^{1-3}$; even higher rates have been reported in the elderly (aged > 65 years $)^{4}$. Polysomnography studies in patients with GAD have revealed sleep abnormalities including problems with sleep latency, early morning insomnia, decrease in total sleep time, and sleep architecture ${ }^{5,6}$.

Epidemiological surveys in primary care have further highlighted that alongside pain, sleep disturbances are the most frequent presenting complaints of patients with GAD and have a strong negative impact on anxiety symptoms and patient functioning ${ }^{7,8}$.

Different classes of agents used for the treatment of GAD have different effects on sleep. While benzodiazepines appear to improve measures of sleep onset and/or sleep maintenance ${ }^{9}$, selective serotonin reuptake inhibitors (SSRIs) tend to have a disruptive effect on sleep. In healthy volunteers, SSRIs, such as paroxetine and fluvoxamine, reduce sleep efficiency and increase the number of awakenings and sleep onset latency ${ }^{10}$. SSRIs have also been reported to reduce the latency and duration of rapid eye movement sleep ${ }^{11}$. There are strong indications that SSRIs might also have sleep-disrupting effects in patients with GAD. It is well established that insomnia is reported as an adverse event with many SSRIs - across anxiety and depression indications, insomnia rates are 9-28\% ${ }^{12-15}$. Insomnia rates reported with the serotoninnorepinephrine reuptake inhibitors (SNRIs) duloxetine and venlafaxine appear to be similar to those reported for SSRIs ${ }^{16,17}$.
Pregabalin, a ligand for the $\alpha 2 \delta$ subunit of voltage-gated calcium channels, has been shown to effectively reduce the symptoms of $\mathrm{GAD}^{18-20}$. It has also demonstrated substantial beneficial effects on sleep. In healthy volunteers, it increases slow-wave sleep as a proportion of both the total sleep period and the duration of stage 4 sleep, and reduces the number of awakenings of more than 1 minute in duration ${ }^{21}$.

A recent trial of pregabalin in $\mathrm{GAD}^{22}$ included subjective assessment of sleep using the Medical Outcomes Study Sleep Scale (MOS-SS). In this study, we fully describe the effects of pregabalin on sleep and further characterize the beneficial effects of pregabalin on frequency and intensity of sleep disturbances in GAD. Using a statistical mediation model, we further sought to ascertain the extent to which pregabalin's effect on sleep disturbance is attributable to a direct effect of the drug, as well as an indirect effect mediated through anxiety symptoms.

\section{Methods}

Data were obtained from a randomized, double-blind, flexible-dose, parallel-group, placebo- and active-controlled study in 394 patients with GAD. The primary endpoint in the study was anxiety symptom change, measured by Hamilton Anxiety Rating Scale (HAM-A) total score, from baseline to the last post-randomization visit prior to dose tapering. The full methodology and results of this trial have been described elsewhere ${ }^{22}$. Briefly, patients were randomized to 8 weeks of flexible-dose treatment with pregabalin (300-600 mg/day), venlafaxine XR (75-225 $\mathrm{mg}$ /day) or placebo. Study participants were male (147/374) and female (227/374) adult outpatients, aged 18-65 years, meeting DSM 
IV-TR criteria for GAD. Further inclusion requirements were a HAM-A total score of $\geq 20$ and a score of $\geq 10$ on both psychic and somatic subscales of HAM-A at screening and baseline visits. Patients with mild depression (Hamilton Depression Rating Scale score of $\leq 14$ ) were allowed to participate in the trial.

This study was conducted in compliance with the ethical principles originating in or derived from the Declaration of Helsinki and in compliance with all International Conference on Harmonization (ICH) Good Clinical Practice (GCP) Guidelines. In addition, all local regulatory requirements were followed, in particular, those affording greater protection to the safety of trial participants. The final protocol, any amendments and informed consent documentation were reviewed and approved by the Institutional Review Board(s) (IRB) and/or Independent Ethics Committee(s) (IEC) at each of the investigational centers participating in the study. The investigators explained the nature, purpose, and risks of the study to each subject, including information regarding probability of receiving a placebo treatment in the study. Each subject was informed that he/she could withdraw from the study at any time and for any reason. Each subject was given sufficient time to consider the implications of the study before deciding whether to participate. Written informed consent was obtained prior to the subject entering the study (before initiation of protocol-specified procedures).

All antidepressant, anxiolytic, antipsychotic, sedative/hypnotic or psycho-active therapy was terminated at least 2 weeks ( 5 weeks for fluoxetine) prior to the baseline visit. If medication was required for the treatment of insomnia during the 1-week wash-out, zopiclone (3.75 mg/night) or zolpidem ( $5 \mathrm{mg} /$ night) could be taken intermittently as needed on two or fewer nights until
3 days before baseline. Prior to start of study treatment, zolpidem was taken by 8 subjects in the pregabalin arm, 6 in the venlafaxine arm and 5 in the placebo arm. Corresponding figures for zopiclone were 8, 7 and 5, respectively. All such medication was prohibited throughout the study.

The subjective assessment of sleep outcomes was evaluated using the MOS-SS, a patient-reported sleep measure that has been shown to be reliable and valid with good overall measurement properties ${ }^{23}$, including in patients with $\mathrm{GAD}^{24}$. MOS-SS consists of 12 items that assess the key constructs of sleep ${ }^{25}$. According to the developer's scoring algorithm (Table 1), MOS-SS scoring yields seven subscales: sleep disturbance (4 items), snoring (1 item), awaken short of breath or with headache (1 item), quantity of sleep (1 item), optimal sleep (1 item), sleep adequacy (2 items), and somnolence $(3 \text { items })^{25}$. Optimal sleep is a dichotomous variable coded as 1 if the patient reported 7 or 8 hours of sleep per night on the quantity of sleep 1-item subscale or 0 if otherwise. Quantity of sleep score relates to the average number of hours slept (0-24). The other five subscales are transformed linearly to a 0-100 scale with higher scores representing more of the sleep concept being measured. In addition, sleep index measures may also be constructed to provide composite scores. The scoring of the MOS-SS in our study follows its standard algorithm, which (along with the scale itself) is available online at http://www.rand.org/health/surveys_tools/m os/mos_sleep.html.

The MOS-SS was administered at baseline, week 4 and week 8 during the trial. At each administration, the recall period was the previous 4 weeks.

In the results reported here, attention is directed predominantly to the sleep disturbance subscale. The items in this scale (trouble 
Table 1

MOS-SS subscales and sleep index evaluated in this study

\begin{tabular}{|c|c|c|}
\hline Subscale & Items & Range \\
\hline \multicolumn{3}{|l|}{ MOS-SS subscales } \\
\hline Sleep disturbance & $\begin{array}{l}\text { Trouble falling asleep } \\
\text { Time to fall asleep } \\
\text { Restlessness } \\
\text { Awaken during sleep }\end{array}$ & $0-100$ \\
\hline Sleep adequacy & $\begin{array}{l}\text { Enough sleep to feel rested } \\
\text { Amount of sleep needed }\end{array}$ & $0-100$ \\
\hline Daytime somnolence & $\begin{array}{l}\text { Feel drowsy during the day } \\
\text { Trouble staying awake during day } \\
\text { Take short naps }\end{array}$ & $0-100$ \\
\hline Snoring & Snore during sleep & $0-100$ \\
\hline Awaken short of breath or with headache & Awaken short of breath or headache & $0-100$ \\
\hline Quantity of sleep & Quantity of sleep & $0-24$ \\
\hline Optimal sleep & Quantity of sleep & 0 or 1 \\
\hline \multicolumn{3}{|l|}{ Sleep Problems Index II } \\
\hline & $\begin{array}{l}\text { Trouble falling asleep } \\
\text { Time to fall asleep } \\
\text { Restlessness } \\
\text { Awaken during sleep } \\
\text { Awaken short of breath or headache } \\
\text { Enough sleep to feel rested } \\
\text { Amount of sleep needed } \\
\text { Feel drowsy during the day } \\
\text { Trouble staying awake during day }\end{array}$ & $0-100$ \\
\hline
\end{tabular}

falling asleep, time to fall asleep, restlessness, and awaken during sleep) are closely related to typical problems experienced by patients with GAD. The broader 9-item MOS-SS Sleep Problems Index II was also examined to capture a range of sleep issues.

\section{Statistical analysis}

All randomized patients with at least one post-baseline assessment were included in the analysis. MOS-SS score changes from baseline to week 4 and week 8 were tested for statistical significance using an analysis of covariance model with main effects of treatment, study center, and baseline score as covariates. Treatment comparisons versus placebo were performed using Dunnett's test.

To characterize the direct and indirect effects of pregabalin and venlafaxine XR treatments on sleep disturbance -the primary aim of this post-hoc analysis- a statistical mediation model was used. Mediation models can be employed to characterize the relationship between an independent variable and a dependent variable through the introduction of one or more mediator variables ${ }^{26,27}$. In our model, we analyzed the effects of pregabalin and venlaflaxine XR (independent variables) on sleep disturbance (dependent 
variable) through the introduction of a mediator variable, notably the effect of anxiety symptoms.

A set of multivariate regression equations were simultaneously fit using full information maximum likelihood to the average of HAM-A total score and MOS-SS sleep disturbance subscale data using PROC MODEL in SAS (SAS Institute Inc, Cary, NC, USA, 2000). Data for all participants were averaged; therefore, every participant with at least one post-baseline data point was included in the modeling. The direct and indirect effects on sleep disturbance were estimated as the percentage of total effect that was explained by the path, identified as paths A, B and C in Figure 1 (paths for venlafaxine XR not shown). Estimates were tested for statistical significance at a nominal alpha level of 0.05 .
To assess the sensitivity of the results relative to missing data, we applied the same mediation model separately to only week 4 data, to only week 8 data, and to week 8 data using last observation carried forward.

All analyses were conducted using SAS version 8.2 (SAS Institute, 2000).

\section{Results}

A total of 374 subjects were randomized to pregabalin $(\mathrm{n}=121)$, venlafaxine XR ( $\mathrm{n}$ $=125)$ or placebo $(\mathrm{n}=128)$ groups. Of these, $71 \%$ completed the study; fewer venlafaxine XR-treated patients completed the study $(67.2 \%)$ compared with pregabalin- or placebo-treated patients $(72.7 \%$ in each group). Patients were predominantly fe-

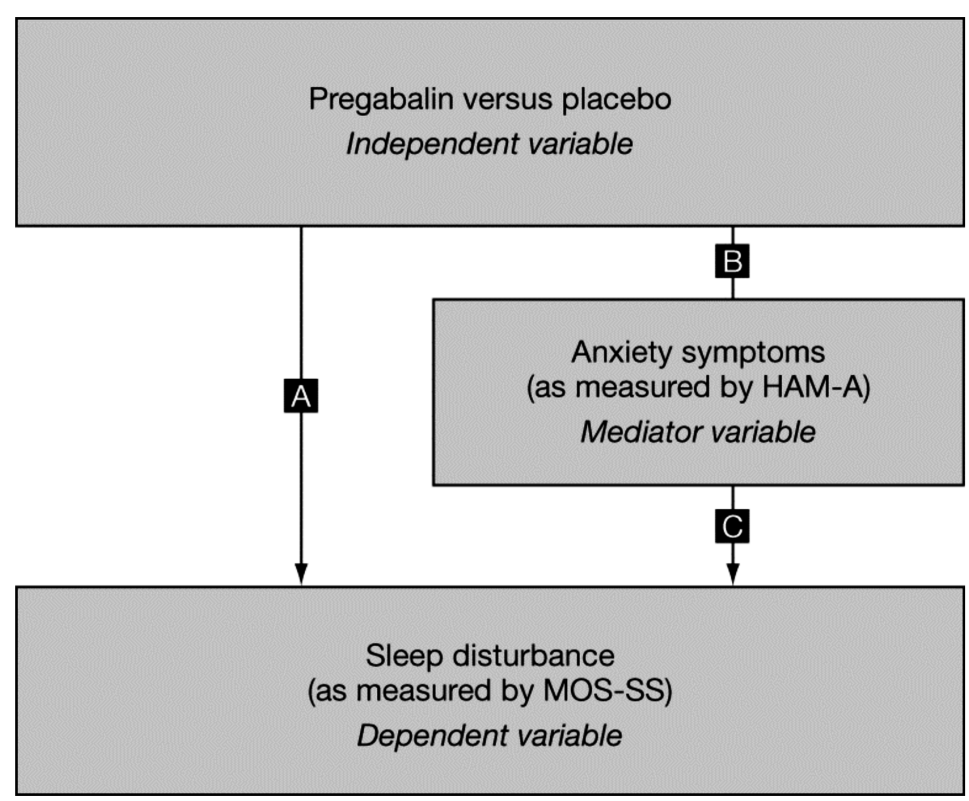

Figure 1. Mediation model illustrating linkages between treatment with pregabalin, HAM-A total score, and MOS-SS sleep disturbance. Path A represents the direct effect of pregabalin on sleep disturbance, while path $\mathrm{B} * \mathrm{C}$ represents the indirect effect 
male, with a mean age of 41 years. The mean duration of GAD diagnosis was approximately 4 years. Demographic characteristics and mean baseline anxiety and sleep disturbance scores were similar in the three groups (Table 2).
Pregabalin showed significant efficacy in reducing scores on the sleep disturbance subscale of MOS-SS at both week 4 and week 8 compared with placebo $(p<0.05)$. Treatment with pregabalin also resulted in significant improvement in awaken short of

Table 2

Demographic and baseline characteristics of subjects randomized to treatment

\begin{tabular}{lccc} 
Baseline characteristic & Pregabalin $\mathrm{n}=121$ & Venlafaxine XR $\mathrm{n}=125$ & Placebo $\mathrm{n}=128$ \\
\hline Age, mean years \pm SD & $39.5 \pm 11.9$ & $42.6 \pm 11.8$ & $40.2 \pm 12.1$ \\
\hline Gender, $\mathrm{n}(\%)$ & $44(36.4)$ & $53(42.4)$ & $50(39.1)$ \\
$\quad$ Male & $77(63.6)$ & $72(57.6)$ & $78(60.9)$ \\
\hline Female & 3.1 & 4.0 & 4.6 \\
\hline Duration of GAD, mean & $0-30.5$ & $0-34.9$ & $0-60.9$ \\
years and range & $27.6 \pm 0.4$ & $27.4 \pm 0.4$ & $26.8 \pm 0.8$ \\
\hline HAM-A total score, mean \pm SD & 56.13 & 53.66 & 51.65 \\
\hline MOS-SS sleep disturbance & $(22.58)$ & $(23.57)$ & $(24.05)$ \\
score, mean (SD) & &
\end{tabular}

breath, sleep adequacy and sleep abnormalities evaluated by the Sleep Problems Index II $(p<0.05$; Table 3$)$. There was a trend towards a detrimental effect of venlafaxine XR on most sleep measures (Table 3). There were no significant differences in the mean change from baseline to weeks 4 and 8 among the three treatment groups for the MOS-SS subscale scores for snoring, quantity of sleep and somnolence.

The mediation model results indicated that $53 \%$ of the total effect on sleep disturbance due to pregabalin treatment (over placebo) was a direct effect $(p=0.0072)$, denoted as path A in Figure 1. The indirect effect, mediated through anxiety symptoms, was estimated as $47 \%(p=0.015)$, path $\mathrm{B} * \mathrm{C}$ in Figure 1 . As patients treated with venlafaxine XR experienced more sleep problems compared with those on placebo, partitioning the total effect into direct and indirect effect resulted in heterogeneous, non-significant effects that could not be interpreted meaningfully.

\section{Discussion}

Results of this study show that pregabalin improves sleep, as the result of two effects that could be indicative of a dual mechanism: a direct treatment effect and an indirect effect via its anxiolytic action. The direct effect of pregabalin on improving sleep is an important finding, given that sleep disturbances are core complaints in patients suffering from $\mathrm{GAD}^{1-3}$ and because of the potential detrimental impact on sleep of many currently available agents ${ }^{12-17}$.

Current data on the impact of anxiolytic drugs on sleep in patients with GAD are limited. Several polysomnography studies have identified negative effects of SSRI/SNRIs, and while benzodiazepines may have sleep benefits their long-term use is not recommended due to dependence potential, and next-day "hangover" effects ${ }^{28}$. Although SSRI/SNRIs have shown significant effica- 


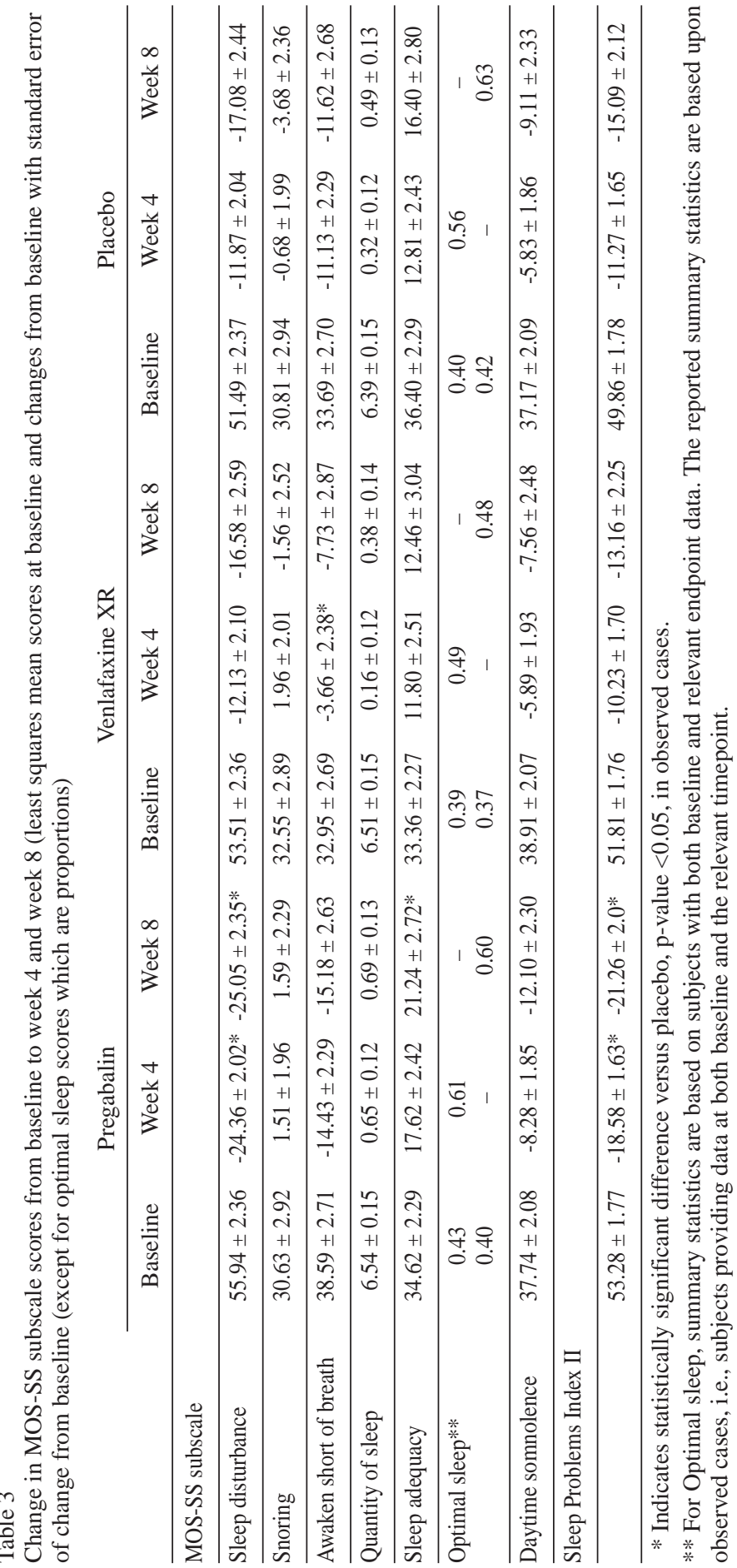


cy in reducing depression and anxiety symptoms, clinical outcomes are likely to be suboptimal for many patients with sleep disturbances ${ }^{29}$. Clinical management of patients should focus on the comprehensive treatment of all anxiety-related symptoms.

Several studies have sought to determine the directional relationship between GAD and insomnia; however, as yet, it is not entirely clear whether GAD predisposes people to develop insomnia or vice versa, or whether it is a bidirectional relationship. The emerging trend appears to indicate that the directionality of association is from anxiety to insomnia ${ }^{30-32}$. What is clear, however, is that patients with GAD are at increased risk of developing sleep-related problems and that patients with chronic insomnia are at increased risk of developing $\mathrm{GAD}^{4,33}$. Mean baseline scores on MOS-SS subscales from our study were consistent with past reports of sleep impairment in GAD. For example, mean baseline sleep disturbance scores ranged from 51.5-56.0 in this study compared with a considerably lower mean score of 24.5 in a survey of the general population $^{23}$. Also in the same survey, mean sleep adequacy was 60.5 , whereas a lower mean score of 34.8 was obtained at baseline across the three groups in this study ${ }^{23}$.

Patients treated with pregabalin showed a significant decrease in MOS-SS sleep disturbance (relating to sleep onset and maintenance) and Sleep Problem Index II subscale scores. The magnitude of change on the sleep disturbance score from baseline to week 4 (-24.36) and from baseline to week $8(-25.05)$ was higher than the estimated threshold value for a clinically important difference of $7.6^{24}$. This shows that improvements are not only statistically significant but are also substantial and thus most likely clinically meaningful.
In our mediation model, we hypothesized that part of the total effect of pregabalin on sleep disturbance is the direct result of treatment itself, not mediated through anxiety symptoms. Readers should interpret the direct effect of treatment as that due to any factor or factors not studied as a mediator in the model. Results showed that the total effect of pregabalin on sleep disturbance can be partitioned into a direct effect (53\%, including factors not accounted for in the mediation model) and an indirect effect mediated through improvement in anxiety symptoms $(47 \%)$. Both effects were statistically significant. In contrast, venlafaxine XR did not appear to have a favorable impact on sleep.

Compared with the main analysis, which averaged available measurements on each subject across the entire study, sensitivity analyses at week 4 only, week 8 only, and week 8 using last observation carried forward revealed a consistent pattern of main findings. For example, the direct effect of pregabalin on sleep disturbance continues to be about $50 \%$ or more, and conversely the indirect effect through anxiety symptoms was about $50 \%$ or less, while these corresponding effects from venlafaxine XR were not present.

Our analysis has several potential limitations. The mediation model is a post-hoc analysis of a secondary endpoint and the sample size was not based on effect sizes observed for the sleep disturbance endpoint. One of the inclusion criteria for the study was that patients must score $\geq 10$ on both psychic and somatic HAM-A subscales. This could have resulted in a study population different from that studied in other clinical trials. Although we included patients with mild depressive symptoms, we excluded those with a current or within the past 6 months diagnosis of major depressive disorder. This and the exclusion of patients with other comorbid disorders could limit the 
generalizability of our results to the clinical GAD population, given the high frequency of co-morbid conditions typically associated with GAD in clinical practice ${ }^{34}$.

\section{Conclusions}

Our study further characterized the beneficial effect of the $\alpha 2 \delta$ ligand pregabalin in reducing sleep disturbances in patients with GAD. The analysis revealed that the total treatment effect on sleep consisted of both direct and indirect (mediated via anxiety symptoms) components, of which the former appeared to be slightly more important.

\section{Acknowledgments}

The authors thank Michael Treglia for his valuable comments during the preparation of the manuscript.

\section{References}

1. Papadimitriou GN, Kerkhofs M, Kempenaers C, Mendlewicz J. EEG sleep studies in patients with generalized anxiety disorder. Psychiatry Res 1988; 26(2): 183-190.

2. Papadimitriou GN, Linkowski P, Kerkhofs M, Kempenaers C, Mendlewicz J. Sleep EEG recordings in generalized anxiety disorder with significant depression. J Affect Disord 1988; 15(2): 113-118.

3. Belanger L, Morin CM, Langlois F, Ladouceur R. Insomnia and generalized anxiety disorder: effects of cognitive behavior therapy for generalized anxiety disorder on insomnia symptoms. J Anxiety Disord 2004; 18(4): 561-571.

4. Taylor DJ, Lichstein KL, Durrence HH, Reidel BW, Bush AJ. Epidemiology of insomnia, depression, and anxiety. Sleep 2005; 28(11): 1457-1464.

5. Saletu-Zyhlarz G, Saletu B, Anderer P, Brandstätter N, Frey R, Gruber G, et al. Nonorganic insomnia in general- ized anxiety disorder. Controlled studies on sleep, awakening and daytime vigilance utilizing polysomnography and EEG mapping. Neuropsychobiology 1997; 36(3): 117-129.

6. Fuller KH, Waters WF, Binks PG, Anderson T. Generalized anxiety and sleep architecture: a polysomnographic investigation. Sleep 1997; 20(5): 370-376.

7. Wittchen HU, Kessler RC, Beesdo K, Krause P, Höfler M, Hoyer J. Generalized anxiety and depression in primary care: prevalence, recognition, and management. J Clin Psychiatry 2002; 63 (Suppl 8): 24-34.

8. Ohayon MM. Prevalence of DSM-IV diagnostic criteria of insomnia: distinguishing insomnia related to mental disorders from sleep disorders. J Psychiatr Res 1997; 31(3): 333-346.

9. Holbrook AM, Crowther R, Lotter A, Cheng C, King D. Meta-analysis of benzodiazepine use in the treatment of insomnia. CMAJ 2000; 162(2): 225-233.

10. Silvestri R, Pace-Schott EF, Gersh T, Stickgold R, Salzman C, Hobson JA. Effects of fluvoxamine and paroxetine on sleep structure in normal subjects: a home-based Nightcap evaluation during drug administration and withdrawal. J Clin Psychiatry 2001; 62(8): 642-652.

11. Saletu B, Frey R, Krupka M, Anderer P, Grünberger J, See WR. Sleep laboratory studies on the single-dose effects of serotonin reuptake inhibitors paroxetine and fluoxetine on human sleep and awakening qualities. Sleep 1991; 14(5): 439-447.

12. Lexapro (escitalopram) FDA approved label. Forest Laboratories, Inc; October 2008. Available at http://www.fda. gov/cder/foi/label/2008/021323s28s29,021365s18s19lbl.pdf Accessed February 2009.

13. Paxil (paroxetine) FDA approved label. GlaxoSmith Kline; October 2008. Available at http://www.fda.gov/cder/foi/ label/2008/020031s060,020936s037,020710s024lbl.pdf Accessed February 2009.

14. Prozac (fluoxetine) FDA approved label. Eli Lilly and Company; June 2008. Available at http://www.fda.gov/cder/ foi/label/2008/018936s086lbl.pdf. Accessed February 2009.

15. Lustral (sertraline) summary of product characteristics. Pfizer Inc.; January 2009. Available at http://emc.medi cines.org.uk/emc/assets/c/html/DisplayDoc.asp?Document ID=1467. Accessed February 2009.

16. Cymbalta (duloxetine) FDA approved label. Eli Lilly and Company; June 2008. Available at http://www.fda.gov/cder/ foi/label/2008/022148lbl.pdf. Accessed February 2009.

17. Effexor (venlafaxine) FDA approved label. Wyeth Pharmaceuticals Inc.; May 2008. Available at http://www.fda.gov/ cder/foi/label/2008/020151s051lbl.pdf. Accessed February 2009. 
18. Feltner DE, Crockatt JG, Dubovsky SJ, Cohn CK, Shrivastava RK, Targum SD, et al. A randomized, doubleblind, placebo-controlled, fixed-dose, multicenter study of pregabalin in patients with generalized anxiety disorder. J Clin Psychopharmacol 2003; 23(3): 240-249.

19. Rickels K, Pollack MH, Feltner DE, Lydiard RB, Zimbroff DL, Bielski RJ, et al. Pregabalin for treatment of generalized anxiety disorder: a 4-week, multicenter, double-blind, placebo-controlled trial of pregabalin and alprazolam. Arch Gen Psychiatry 2005; 62(9): 1022-1030.

20. Feltner D, Wittchen HU, Kavoussi R, Brock J, Baldinetti F, Pande AC. Long-term efficacy of pregabalin in generalized anxiety disorder. Int Clin Psychopharmacol 2008; 23(1): 18-28.

21. Hindmarch I, Dawson J, Stanley N. A double-blind study in healthy volunteers to assess the effects on sleep of pregabalin compared with alprazolam and placebo. Sleep 2005; 28(2): 187-193.

22. Kasper S, Herman B, Nivoli G, Van Ameringen M, Petralia A, Mandel FS, et al. Efficacy of pregabalin and venlafaxine-XR in generalized anxiety disorder: results of a double-blind, placebo-controlled 8-week trial. Int Clin Psychopharmacol 2009; 24(2): 87-96.

23. Hays RD, Martin SA, Sesti AM, Spritzer KL. Psychometric properties of the Medical Outcomes Study Sleep measure. Sleep Med 2005; 6(1): 41-44.

24. Bollu V, Bushmakin AG, Cappelleri JC. Psychometric evaluation of the Medical Outcomes Study Sleep Scale in patients with generalized anxiety disorder. 2008 International Society for Quality of Life Research meeting abstracts. Quality of Life Research 2008; 17(Suppl): A114 (abstract 1591). Available at http://www.isoqol.org/2008_ conference/AbstractsForBooklet_2008v3.pdf

25. Hays RD, Stewart AL. Sleep measures. In: Stewart AL, Ware JE Jr, eds. Measuring Function and Well-being. Durham, NC: Duke University Press 1992; 235-259.
26. MacKinnon DP. Introduction to Statistical Mediation Analysis. New York, NY: Lawrence Erlbaum Associates; 2008.

27. Iacobucci D. Mediation Analysis. Los Angeles, CA: Sage; 2008.

28. Roth T, Roehrs T. A review of the safety profiles of the benzodiazepine hypnotics. J Clin Psychiatry 1991; 52(Suppl): 38-41.

29. Pollack M, Kinrys G, Krystal A, McCall WV, Roth T, Schaefer K, et al. Eszopiclone coadministered with escitalopram in patients with insomnia and comorbid generalized anxiety disorder. Arch Gen Psychiatry 2008; 65(5): 551-562.

30. Johnson EO, Roth T, Breslau N. The association of insomnia with anxiety disorders and depression: exploration of the direction of risk. J Psychiatr Res 2006; 40(8): 700-708.

31. Ohayon MM, Roth T. Place of chronic insomnia in the course of depressive and anxiety disorders. J Psychiatr Res 2003; 37(1): 9-15.

32. Jansson-Fröjmark M, Lindblom K. A bidirectional relationship between anxiety and depression, and insomnia? A prospective study in the general population. J Psychosom Res 2008; 64(4): 443-449.

33. Neckelmann D, Mykletun A, Dahl AA. Chronic insomnia as a risk factor for developing anxiety and depression. Sleep 2007; 30(7): 873-880.

34. Stein DJ. Comorbidity in generalized anxiety disorder; impact and implications. J Clin Psychiatry 2001; 62 (Suppl 11): 29-34.

Address for correspondence:

Joseph C. Cappelleri

Pfizer Inc. 50 Pequot Avenue

New London

CT 06320, USA

Phone: + 1 (860) 732-8668

E-mail: joseph.c.cappelleri@pfizer.com

\section{Disclosures}

This study was supported by Pfizer Inc. Vamsi Bollu was an employee of Pfizer Inc. at the time of the preparation of this manuscript. Editorial assistance with the preparation of this manuscript was provided by Azita Tofighy, $\mathrm{PhD}$, medical writer at GCL, and funded by Pfizer Inc.

Clinical trial registration number NCT00151450 